\title{
Compósito trifásico baseado em resíduo de goma de mascar-sbr e partículas de quartzo
}

\author{
Three-phase composite based on Chewing \\ gum-SBR waste and quartz particles
}

\begin{abstract}
Fabíola da Silveira Maranhão ${ }^{1}$, Daniela Marques do Nascimento ${ }^{1}$, Fernando Gomes de Souza Junior ${ }^{1,2}$, Thuanny Moraes de Almeida ${ }^{1}$,Thiago Castro Lopes ${ }^{1}$, Tamara Nunes da Cunha Moreira ${ }^{3}$, Leila Y. Visconte ${ }^{1}$, Romildo Dias Toledo Filho ${ }^{2,3}$
\end{abstract}

\footnotetext{
${ }^{1}$ Laboratório de Biopolímeros e Sensores / Instituto de Macromoléculas / Universidade Federal do Rio de Janeiro, Instituto de Macromoléculas Professora Eloisa Mano, Av. Horácio Macedo, nº 2030, Bloco J, CEP: 21941-598, Rio de Janeiro, Cidade Universitária, Brasil.

${ }^{2}$ Programa de Engenharia de Nanotecnologia - COPPE/Universidade Federal do Rio de Janeiro, Programa de Engenharia de Nanotecnologia, Rua Moniz Aragão n 360, Bloco 1, CEP: 21941-598, Rio de Janeiro, Cidade Universitária, Brasil. ${ }^{3}$ Núcleo de Ensino e Pesquisa em Materiais e Tecnologias de Baixo Impacto Ambiental na Construção SustentávelNUMATS- POLI/COPPE/Universidade Federal do Rio de Janeiro, Núcleo de Ensino e Pesquisa em Materiais e Tecnologias de Baixo Impacto Ambiental na Construção Sustentável- NUMATS- POLI/COPPE/UFRJ, Rua Moniz Aragão ${ }^{\circ}$ 360, Bloco 1, CEP: 21941-598, Rio de Janeiro, Cidade Universitária, Brasil.

e-mail: fgsj@ufrj.br
}

\section{RESUMO}

O elevado consumo de produtos industrializados e o seu rápido descarte produzem grandes quantidades de resíduos sólidos, dos quais, os principais constituintes são os materiais poliméricos. Dentre estes, as borrachas de butadieno estireno merecem destaque. Esse material é usado em diversos setores, entre eles, o alimentício, para produção de chicles. A reciclagem desses materiais deve ser sempre pesquisada, buscando, principalmente, novas aplicações. Assim, este trabalho usou a borracha residual (BP) como matriz para cristais de quartzo. Os materiais preparados foram estudados por diversas técnicas, como, por exemplo, a Análise Termogravimétrica (TGA) e a Espectroscopia no Infravermelho por Transformada de Fourier (FTIR). Além disso, os materiais tiveram suas propriedades elétricas medidas. Foram feitas medidas de capacitância, campo elétrico, resistividade e densidade de corrente. Entre os materiais testados, o compósito carregado com 44,4\% de quartzo apresentou a resposta mais linear e mais intensa de variação de carga elétrica em função da pressão aplicada. Portanto, os resultados mostraram que foram obtidos materiais com potencial aplicação no campo de sensores capacitivos de pressão.

Palavras-Chave: SBR, goma de mascar, partículas de quartzo, sensor

\section{ABSTRACT}

The high consumption of industrialized products and their disposal produce large quantities of solid waste, of which the principal constituents are polymeric materials. Among these, the butadiene-styrene rubbers deserve special mention. This material is used in various industries, including food, for the production of chewing gum. Thus, the recycling of these materials must always be researched, mainly seeking new applications. Therefore, this work used residual rubber (BP) as a matrix for quartz crystals. The materials prepared were studied by several techniques, such as Thermogravimetric Analysis (TGA) and Fourier Transform Infrared Spectroscopy (FTIR). Besides, the materials also had their electrical properties measured. Capacitance, electric field, resistivity and current density measurements were performed. Among the tested materials, the composite loaded with $44.4 \%$ of quartz presented the most linear and intense response of electric charge variation as a function of the applied pressure. Therefore, the obtained results allow proving that were accomplished materials with the potential of application in the field of capacitive pressure sensors.

Keywords: SBR, chewinggum, quartzparticles, sensor. 


\section{INTRODUÇÃO}

O elevado volume de resíduos sólidos urbanos e industriais é uma das grandes preocupações deste século, uma vez que a industrialização aumentou consideravelmente a quantidade de resíduos. Estes, muitas vezes, têm destinação inadequada ou não aceitável do ponto de vista econômico e ambiental [1].

Os elastômeros são polímeros usados para a produção de pneus, calçados e chicles, os quais, muitas vezes, são descartados de maneira inadequada e causam danos ao ambiente. Estes últimos são obtidos a partir de um polímero amorfo, oriundo da borracha de estireno butadieno (SBR). Contudo, para a fabricação de outros artefatos, como pneus e calçados, as borrachas passam por uma etapa denominada vulcanização que confere às cadeias reticulações com enxofre, o que aumenta a resistência à degradação desses materiais [2, 3]. Visando a reutilização desses compostos, é possível observar na literatura o uso da borracha de estirenobutadieno pós-consumo na produção de compósitos leves [4] e, também em matrizes de poliestireno para aumento da tenacidade [5]. Já a reciclagem é realizada a partir do uso de micro-ondas em que o aquecimento favorece a quebra das ligações cruzadas e ocorre um processo conhecido como desvulcanização [6].

Por sua vez, há a borracha SBR que não passa pelo processo de vulcanização que é empregada no setor alimentício para a produção dos chicles. Seu uso está relacionado ao baixo custo e facilidade de processamento. Ainda que não passe pelo processo de vulcanização que confere maior resistência a degradação, a sua decomposição leva mais de 5 anos. Esse tempo é suficiente para causar poluição visual e principalmente, permitir que animais se alimentem e morram por asfixia [6-7], isso ocorre, pois, a goma mascada passa a se comportar de forma pegajosa, grudando nos bicos ou focinhos dos animais. Anualmente, são consumidas 1,74 trilhões de goma de mascar, desse consumo 90\% são descartadas [8].

Diante disso, torna-se importante a reciclagem desse material. Uma das alternativas foi mostrada pela empresa GumPoint, que dispõe de lixeiras para descarte de gomas mastigadas. Estas, após a coleta, são usadas para a produção de solados, copos, botas de borracha e chinelos [9]. Porém, pouco há na literatura sobre a recuperação da goma de mascar, embora já existam alguns trabalhos pioneiros sobre o seu uso para a produção de materiais termoplásticos e sensores capacitivos. Estes sensores são capazes de medir a variação de pressão através da aplicação de forças externas [10].

O quartzo é um dos minerais mais abundantes da crosta terrestre, sua composição é sílica e oxigênio $\left(\mathrm{SiO}_{2}\right)$. Sua estrutura cristalina conhecida como quartzo $\alpha$, foi a principal para descoberta de vários fenômenos físicos como a piroeletricidade e a piezoeletricidade, a primeira relacionada a capacidade de fornecer um potencial elétrico temporário quando aquecidos e a segunda relacionada a geração de potencial elétrico ao sofrer alguma pressão mecânica. Essas funcionalidades são atrativas e a literatura mostra a produção de compósitos com quartzo para geração de energia elétrica ou para o monitoramento da pressão interna de poços de petróleo [11]. Já as borrachas SBRsão isolantes, o que dificulta a condutividade elétrica, porém é possível torná-la condutora através da modificação da matriz. Por exemplo, Liu e colaboradores aumentaram a condutividade do SBR de $2 \times 10^{-14} \mathrm{~S} / \mathrm{cm}$ para $3,8 \times 10^{-1} \mathrm{~S} / \mathrm{cm}$, via inserção de nanotubos de carbono [12].

Assim, o presente trabalho tem o objetivo de avaliar o reaproveitamento do SBR consumido como chicle e seu potencial uso como sensor de pressão, quando carregado com sílica e vulcanizado. Esse material pode ser usado como pavimento inteligente para edificações, onde poderia detectar a presença de pessoas, alterado, por um sistema de controle central, a luminosidade - via acendimento de lâmpadas - ou mesmo a temperatura - via acionamento de sistema de refrigeração. Desta forma, aqui foram preparadas misturas de SBR virgem com chicles, que foram usadas como base para o preparo de um compósito carregado com quartzo. Estes materiais, foram caracterizados por diversas técnicas, como a Análise Termogravimétrica (TGA), a Espectroscopia no Infravermelho por Transformada de Fourier (FTIR) e por caracterizações elétricas. Estas últimas permitiram inferir que os materiais preparados apresentaram variação linear da capacitância em função da pressão aplicada, podendo ser úteis como sensores capacitivos de pressão.

\section{MATERIAIS E MÉTODOS}

\subsection{Materiais}

Os materiais foram usados na forma em que foram recebidos. O SBR comercial foi fornecido pela Empresa LANXESS com as seguintes especificações: viscosidade Mooney $\left(1+4 @ 100^{\circ} \mathrm{C}\right)$ igual a 49 e teor de Estireno de 23\%. A Goma de Mascar foi obtida de um fabricante nacional. Os autores podem revelar a origem do material em caso de justa necessidade. O Cristal de Quartzo foi fornecido pela Empresa Cristais Aquarius, com 99\% de pureza, opaco e brilhoso. Esse material foi moído em um moinho de bolas e peneirado para obtenção das partículas com tamanho $425 \mu \mathrm{m}$. Foi usado o sistema de vulcanização, composto por ácido esteárico P.A doado pela Teadit, óxido de zinco P.A fornecido pela Vetec, enxofre P.A 
fornecido pela Uniroyal e acelerador TMTD doado pela Teadit, seguindo a formulação apresentada na norma ASTM D-3185/88 tópico 5.

\subsection{Equipamentos}

Os equipamentos usados foram: Moinho de Bolas Ultracentrífugo da marca RETSCH ZM 200, localizado no Laboratório de Caracterização Tecnológica, Centro de Tecnologia Mineral (CETEM). O Analisador Termogravimétrico (TGA), modelo STA 6000 , usando atmosfera de nitrogênio, na faixa de temperatura de $30^{\circ} \mathrm{C}$ $700^{\circ} \mathrm{C}$ com taxa de aquecimento de $20^{\circ} \mathrm{C}$ por minuto, localizado no Laboratório de Apoio Instrumental 3 (IMA-UFRJ). O Espectrômetro de Infravermelho com Transformada de Fourier (FTIR), modelo NicoletiN10 foi feito usando acessório de reflexão total atenuada, faixa de análise de 4000 a $650 \mathrm{~cm}^{-1}$ com número de scans de 132 e resolução de $4 \mathrm{~cm}^{-1}$, localizado no Laboratório de Apoio Instrumental 3 (IMA-UFRJ), O Eletrômetro da marca Keithley 6517B, localizado no Laboratório de Apoio Instrumental 3 (IMA-UFRJ). O Misturador de Rolos da marca LAB TECH EngineeringCompany LTD, localizado no Laboratório de Apoio Técnico 1 (IMA-UFRJ). O RPA 2000 (Rubber ProcessAnalyser) da marca Alpha Technologies, a $160{ }^{\circ} \mathrm{C}$ por 1h, localizado no Laboratório de Apoio Técnico 2 (IMA-UFRJ). A Prensa Hidráulica da marca CARVER, prensa de 12 toneladas, localizada no Laboratório de Compostos de Borracha (IMA-UFRJ).

\subsection{MÉTODOS}

\subsubsection{Preparo dos Compósitos a partir da Goma Residual:}

O quartzo foi triturado com o auxílio de um Moinho de Bolas e peneirado até diâmetro máximo de $425 \mu \mathrm{m}$. Os compósitos foram preparados usando a borracha SBR (Borracha de butadieno-estireno); a goma mascada, denominada BP (Borracha pós-processamento); e quartzo nas proporções de 16,6\%, 28,5\%, 37,5\% e 44,4\% $\mathrm{m} / \mathrm{m}$. Essas quantidades foram escolhidas de modo a obter o limiar de percolação das amostras, que depende da quantidade de carga condutora na matriz [13]. As misturas foram preparadas seguindo a norma ASTM D3185/88 tópico 5, que trata da formulação para borracha SBR. Entretanto, foram feitas algumas adaptações, seguindotrês etapas:

Primeira etapa: A primeira etapa consistiu na mistura de $50 \mathrm{phr}$ da borracha SBR com os agentes de vulcanização e metade da quantidade de quartzo, com mistura de duração de 16 minutos, velocidade dos rolos $10 / 15 \mathrm{rpm}$, temperatura a $30^{\circ} \mathrm{C}$ e abertura entre os rolos de $0,30 \mathrm{~mm}$.

Tabela 1: Etapa 1-Concentrações de borracha SBR virgem, quartzo.

\begin{tabular}{c|c|c|c}
\hline Etapa 1- Compósitos & SBR & Quartzo (\%) & Agentes de Vulcanização \\
\hline SBR-BP & & 0 & \\
1 & & 8,30 & ASTM \\
2 & $50 \mathrm{phr}$ & 15,25 & $\mathrm{D}-3185 / 88$ \\
3 & & 18,75 & \\
4 & & 22,20 & \\
\hline
\end{tabular}

Segunda etapa: Esta etapa consistiu na mistura de $50 \mathrm{phr}$ da borracha BP com a outra metade de quartzo, com duração de 7 minutos de mistura, a velocidade dos rolos foi de 10/10 rpm, abertura entre os rolos de $1,64 \mathrm{~mm}$ e temperatura de $30^{\circ} \mathrm{C}$. 
Tabela 2: Etapa 2-Concentrações de goma de mascar, quartzo.

\begin{tabular}{c|c|c}
\hline Etapa 2- Compósitos & Goma de mascar & Quartzo (\%) \\
\hline SBR-BP & & 0 \\
1 & & 8,30 \\
2 & $50 \mathrm{phr}$ & 15,25 \\
3 & & 18,75 \\
4 & & 22,20 \\
\hline
\end{tabular}

Terceira etapa: A última etapa consistiu em juntar as misturas previamente feitas. Esta etapa durou cerca de 4 minutos, seguindo os mesmos procedimentos usados anteriormente.

As amostras preparadas foram analisadas via RPA (Rubber ProcessingAnalyser) da marca Alpha Technologies, para obtenção dos resultados de tempo de cura $\left(\mathrm{t}_{90}\right)$, numa varredura a $160{ }^{\circ} \mathrm{C}$ por 1 hora. Conhecido o $t_{90}$, as amostras seguiram para vulcanização. A vulcanização dos compósitos foi feita em uma prensa hidráulica. Devido à espessura $(0,6 \mathrm{~cm})$ das amostras, foi acrescentado um tempo extra ao processo para assegurar que a vulcanização fosse completa. Assim, as amostras foram vulcanizadas a $160{ }^{\circ} \mathrm{C}$ por um tempo de 7,5 minutos. Este tempo equivale ao $t_{90}$ médio acrescido de 5 minutos, conforme discutido em um trabalho anterior do grupo [14].

\subsubsection{Propriedades Reométricas}

As amostras foram analisadas no RPA (Rubber ProcessingAnalyser), usando a varredura de $160{ }^{\circ} \mathrm{C}$ por 1 hora, nessa análise foi possível obter o t 90 que é o tempo de vulcanização das amostras, MI que é o torque mínimo, Mh que é o torque máximo e o Mh-MI que é a variação dos torques máximo e mínimo.Usando o Programa QtiPlot, foi feita a regressão linear a partir dos valores obtidos e extraídos os coeficientes angulares, lineares e coeficiente de determinação. Os limites de confiança com $95 \%$ de probabilidade $\left(\operatorname{Lc}_{95 \%}\right)$, foram calculados usando a Equação 1,

$L c_{95 \%}=\frac{\operatorname{DesvPad} * K s}{\operatorname{raiz}(n)}(1)$

onde DesvPad é o desvio padrão; Ks é a contante da tabela T de Student com $95 \%$ de probabilidade; e n é a quantidade de valores.

Os resultados são mostrados na Tabela 3 .

\subsubsection{Propriedades Elétricas:}

Foram avaliadas as propriedades elétricas das amostras, seguindo a metodologia empregada por nosso grupo de pesquisa, descrita em um trabalho anterior [15]. O compósito foi colocado no porta-amostra e as placas metálicas (eletrodos) foram sobrepostos nas faces da amostra, a fim de obter a resposta elétrica do material. Estas placas foram acopladas ao cabo triaxial do eletrômetro. Esse arranjo foi colocado sobre uma célula de carga de $25 \mathrm{kN}$, que foi responsável por medir a pressão aplicada e, posteriormente, foram aplicadas diferentes pressões ao material com o auxílio de uma prensa manual Tecniq. As pressões aplicadas foram iguais a 0 ; 0,189; 0,395 e 0,765 MPa. Nesse sentido, para medição da condutividade foi estabelecida a corrente de 100 $\mathrm{V}$, considerando a capacidade do material em receber a energia aplicada e o tempo de teste foi de 2 minutos. A partir desse método, foi possível, com o auxílio de um eletrômetro Keithley 6517B, obter as informações do armazenamento de carga, da diferença de potencial, da resistividade e da densidade corrente elétrica em função da pressão aplicada e do tempo experimental.

\subsubsection{Análise Estatística das Respostas Elétricas.}

Foram obtidos valores de armazenamento de carga $(\mathrm{pF})$, campo elétrico $(\mathrm{E})$, resistividade $(\rho)$ e densidade de corrente (J). A partir desses valores, foi usada a linguagem de programação R para a análise dos dados de (i) tempo de experimento, (ii) quantidade de quartzo, e (iii) pressão aplicada nos materiais. Foram obtidos dados da correlação entre as matrizes, bem como a determinação os valores estatisticamente significantes, com 95\% de probabilidade, esses parâmetros estão relacionados a modelagem realizada [16]. Em seguida, foram feitos gráficos de superfícies de resposta, que facilitaram o entendimento das relações entre as variáveis de entrada (tempo de experimento, quantidade de quartzo e pressão aplicada) e as de saída (armazenamento de carga $(\mathrm{pF})$, campo elétrico $(\mathrm{E})$, resistividade $(\rho)$ e densidade de corrente $(\mathrm{J}))$. 


\section{RESULTADOS E DISCUSSÃO}

A determinação da maneira ideal para se trabalhar o resíduo aqui usado foi etapa chave do trabalho. A goma de mascar é um material pegajoso que consegue aderir em diferentes superfícies. Isso dificulta muito o seu uso. Assim, foi necessário adicionar SBR virgem na proporção de $50 \mathrm{phr}$ e $50 \mathrm{phr}$ de goma residual. As misturas foram feitas a $30{ }^{\circ} \mathrm{C}$. Temperaturas maiores produziram uma indesejada aderência da goma nos rolos. Em seguida, os materiais foram levados para o RPA, que forneceu os valores de tempo ótimo de cura necessário para que a borracha adquira $90 \%$ das ligações cruzadas $\left(\mathrm{t}_{90}\right)$. Também foram obtidos valores do tempo de pré-vulcanização (ts1). Além desses, foram obtidos valores de torque mínimo (MI) que indica a viscosidade da composição não vulcanizada; de torque máximo (Mh), relacionado à densidade de ligações cruzadas; e da variação dos torques máximo e mínimo (Mh-MI) [17]. Esses valores são mostrados na Tabela 3.

Tabela 3: Propriedades reométricas dos compósitos.

\begin{tabular}{c|c|c|c|c|c}
\hline $\begin{array}{c}\text { Quartzo } \\
(\%)\end{array}$ & $\begin{array}{c}\mathbf{t}_{\mathbf{9 0}} \\
\text { (minutos) }\end{array}$ & $\begin{array}{c}\text { ts1 } \\
\text { (minutos) }\end{array}$ & $\begin{array}{c}\text { MI } \\
(\mathbf{d N m})\end{array}$ & $\begin{array}{c}\mathbf{M h} \\
(\mathbf{d N m})\end{array}$ & $\begin{array}{c}\text { Mh-MI } \\
(\mathbf{d N m})\end{array}$ \\
\hline SBR-BP & 2,7 & 1,3 & 0,3 & 9,4 & 9,0 \\
\hline 16,6 & 2,4 & 1,1 & 0,4 & 10,6 & 10,2 \\
\hline 28,5 & 2,2 & 1,0 & 0,4 & 12,3 & 12,0 \\
\hline 37,5 & 2,5 & 1,0 & 0,5 & 14,0 & 13,3 \\
\hline 44,4 & 2,4 & 1,0 & 0,5 & 13,4 & 13,0 \\
\hline Coeficiente angular & $-5,67 \times 10^{-3}$ & $-6,86 \times 10^{-3}$ & $4,56 \times 10^{-3}$ & $1,05 \times 10^{-1}$ & $1,02 \times 10^{-1}$ \\
\hline Coeficiente linear & 2,58 & 1,25 & 0,30 & 9,27 & 8,90 \\
\hline $\mathrm{R}^{2}$ & 0,30 & 0,86 & 0,92 & 0,93 & 0,95 \\
\hline$p$ & 0,337 & 0,024 & 0,009 & 0,008 & 0,005 \\
\hline
\end{tabular}

$\mathrm{R}^{2}$ : Coeficiente de determinação; $\mathrm{p}$ : Grau de confiança de $95 \%(\mathrm{p}<0,05)$.

As modelagens lineares apresentadas na Tabela 3 são estatisticamente relevantes quando $p<0,05$. Valores de $\mathrm{p}$ menores que 0,05 foram encontrados para todos os parâmetros estudados, exceto para $\mathrm{o} \mathrm{t}_{90}$. Ainda assim, os dados mostrados na Tabela 3 permitiram inferir que os valores do tempo ótimo de cura são similares para todos os compósitos. Por sua vez, os baixos coeficientes angulares de ts1 e de MI permitem afirmar que essas propriedades se mantêm constantes ao longo da faixa de teores de quartzo, aqui estudada. Por outro lado, os valores de Mh e MI aumentaram de forma linear com o aumento da quantidade de quartzo $\left(\mathrm{R}^{2}=0,93\right.$ e 0,95 , respectivamente). Isso é atribuído ao aumento da resistência mecânica da matriz polimérica, fruto de quantidades crescentes do quartzo. Sendo assim, o quartzo influenciou nas propriedades viscoelásticas da borracha por ser um material com elevada dureza, isso favorece um efeito hidrodinâmico e a diminuição da viscosidade [18].

Os espectros FTIR dos materiais são mostrados na Figura 1. Além da manutenção de todas as principais bandas do sistema polimérico nos materiais pós-processamento, o uso do FTIR provou a presença do quartzo na matriz polimérica. Os espectros de FTIR mostraram bandas características do polímero butadieno-estireno em 2920 e $2850 \mathrm{~cm}^{-1}$ atribuídas ao alongamento $\mathrm{CH}$ de anéis aromáticos do estireno. O pico em $1600 \mathrm{~cm}^{-1}$ é referente à vibração de estiramento do $\mathrm{CH}_{2} \mathrm{e} \mathrm{CH}_{3}$. Por sua vez, os picos ao redor de 968 e $699 \mathrm{~cm}^{-1}$ foram atribuídos à presença dos isômeros 1,4-transbutadieno e 1,4-cisbutadieno, respectivamente. Ainda há a banda a $3450 \mathrm{~cm}^{-1}$, característica do grupamento $\mathrm{OH}$ da água [19]. Além dessas, nos compósitosexistiu uma banda ao redor de $1060 \mathrm{~cm}^{-1}$, relacionada ao estiramento do grupo funcional $\mathrm{SiO}_{2}$; outra em $842 \mathrm{~cm}^{-1}$, referente à deformação angular do $\mathrm{SiO}_{2}$; e uma última em $450 \mathrm{~cm}^{-1}$, característica das vibrações do grupamento $\mathrm{SiO}_{2}[20]$. 


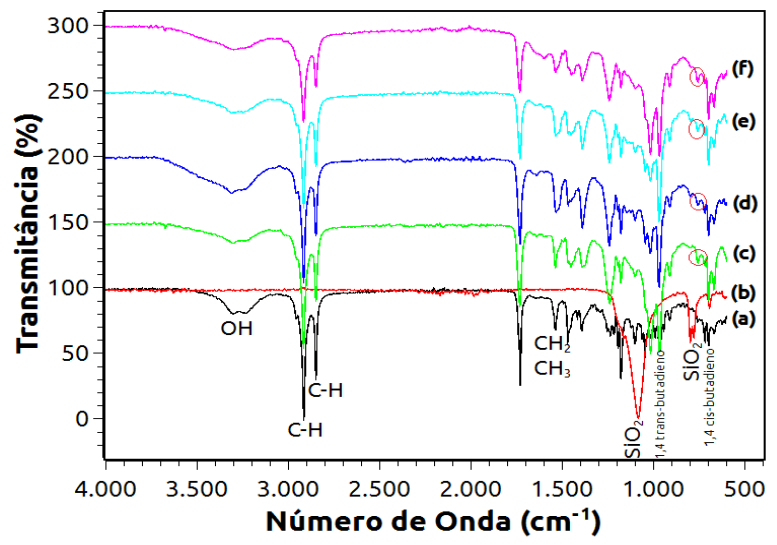

Figura 1: FTIR dos compósitos contendo (a) SBR-BP de Quartzo; (b) 100\% de carga Quartzo; (c) 16,6\% de carga Quartzo; (d) 28,5\% de carga Quartzo; (e) 37,5\% de carga Quartzo e (f) 44,4\% de Quartzo.

A Figura 2 mostra as curvas derivadas das análises termogravimétricas dos compósitos. Esta técnica foi usada com o objetivo de avaliar a influência da inserção da carga inorgânica sobre a resistência térmica dos materiais. Os termogramas mostram picos referentes às degradações dos compostos de poli (butadienoestireno), os quais são identificados em temperaturas acima de $300{ }^{\circ} \mathrm{C}$ e $400{ }^{\circ} \mathrm{C}$ [21]. Especificamente, os termogramas permitiram identificar as primeiras temperaturas de degradação, relacionadas à despolimerização dos segmentos polibutadiênicos da borracha SBR [22], cujos osvalores foram iguais a 335 ${ }^{\circ} \mathrm{C}, 341{ }^{\circ} \mathrm{C}, 349{ }^{\circ} \mathrm{C}, 352{ }^{\circ} \mathrm{C}$ e $352{ }^{\circ} \mathrm{C}$ para os materiais carregados com SBR-BP, 16,6\%, 28,5\%, 37,5\% e $44,4 \%$ de quartzo, respectivamente.

Por sua vez, o segundo e mais relevante evento de degradação, resultante da degradação simultânea do estireno e do butadieno, ocorreu nas temperaturas de $441{ }^{\circ} \mathrm{C}, 449{ }^{\circ} \mathrm{C}, 454{ }^{\circ} \mathrm{C}, 456{ }^{\circ} \mathrm{C}$ e $457{ }^{\circ} \mathrm{C}$ para os materiais carregados com SBR-BP, 16,6\%, 28,5\%, 37,5\% e 44,4\% de quartzo, respectivamente. Assim, fica evidente que quantidades crescentes de quartzo produzem um aumento da resistência térmica do material, tornando-os mais resistentes à degradação. Além disso, há uma contínua diminuição da área dos picos, decorrente do aumento da fração de quartzo, o qual é inerte ao processo de aquecimento. Isso é demonstrado na Figura 2(f).

Além dessas temperaturas de degradação, foi possível observar que, para a amostra SBR-BP houve degradação em torno de $550^{\circ} \mathrm{C}$ referente à pirólise do material, com a consequente produção da mistura dos gases metano, etano, propano/buteno, butano/buteno [22]. Com isso, verificou-se que os materiais são termicamente resistentes até mais de $150^{\circ} \mathrm{C}$, o que abre margem para o uso em temperaturas superiores ao ambiente convencional.

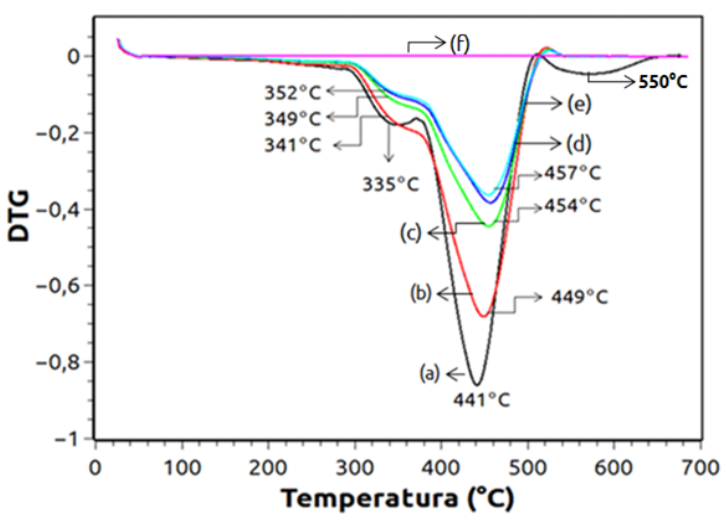

Figura 2: Curvas da derivada da perda de massa em função da temperatura dos compósitos contendo (a) SBR-BP de carga Quartzo; (b) 16,6\% de carga Quartzo; (c) 28,5\% de carga Quartzo; (d) 37,5\% de carga Quartzo; (e) 44,4\% de carga Quartzo e (f) $100 \%$ de carga Quartzo. 
Os diferentes resultados obtidos nas análises da resposta elétrica dos compósitos, sendo esses, armazenamento de carga $(\mathrm{pF})$, campo elétrico $(\mathrm{E})$, resistividade $(\rho)$ e densidade de corrente $(\mathrm{J})$, foram usados para desenvolver as análises estatísticas. Nessas, foram usados os valores iniciais e finais do tempo dos testes elétricos, mostrados na Tabela 4 e assim, os compósitos analisados foram submetidos ao efeito de três variáveis de entrada: (i) diferentes quantidades de quartzo inseridas na matriz polimérica; (ii) pressões de 0; 0,189; 0,395 e 0,765 MPa; e (iii) o tempo de análise, até 2 minutos para cada valor de pressão. Para um maior entendimento acerca da influência dessas variáveis de entrada sobre as de saída (propriedades elétricas) fezse necessário o uso da análise estatística.

A primeira etapa da análise foi a obtenção de uma tabela de correlações entre as variáveis, mostrada na Tabela 5. As variáveis marcadas em negrito são aquelas que mostram as correlações estatisticamente relevantes $(\mathrm{p}<0,05)$ entre as variáveis de entrada e saída [23]. Assim, foi possível inferir que a quantidade de quartzo e a resistividade são inversamente correlacionadas. Portanto, o aumento gradual da quantidade de quartzo produz a diminuição gradual da resistividade. Seguindo com a análise, o tempo está diretamente correlacionado com o armazenamento de carga e com o campo elétrico. A quantidade de quartzo está diretamente correlacionada com o armazenamento de carga e com a densidade de corrente elétrica, porém é inversamente proporcional ao campo elétrico e a resistividade, permitindo concluir que, à medida que há o aumento da carga na matriz polimérica, ocorre o aumento da densidade de corrente e da capacidade do material em armazenar carga elétrica. Isso, consequentemente, gera a diminuição do campo elétrico e da resistividade, o que está de acordo com a Lei de Ohm. Por sua vez, observa-se que a pressão (MPa) está diretamente correlacionada com o armazenamento de carga elétrica e com o campo elétrico. Finalmente, é possível inferir que a carga elétrica acumulada é diretamente relacionada com a quantidade de quartzo e com o tempo de experimento.

Tabela 4: Valores iniciais e finais dos testes nos compósitos, variáveis de entrada e saída.

\begin{tabular}{|c|c|c|c|c|c|c|}
\hline \multicolumn{3}{|c|}{ Variáveis de entrada } & \multicolumn{4}{|c|}{ Variáveis de saída } \\
\hline Tempo (h) & Pressão (Mpa) & Quartzo (\%) & Carga (pF) & $\mathrm{E}(\mathrm{V} . \mathrm{cm})$ & $\rho(\Omega . c m)$ & $\mathrm{J}(\mathrm{A} / \mathrm{cm} 2)$ \\
\hline 0,000 & 0,000 & 0 & $6,02 \mathrm{E}-04$ & $9,10 \mathrm{E}-02$ & $4,96 \mathrm{E}+11$ & $2,01 \mathrm{E}-10$ \\
\hline 0,188 & 0,765 & 0 & 4,77E-03 & $4,34 \mathrm{E}+00$ & $5,20 \mathrm{E}+11$ & $1,98 \mathrm{E}-10$ \\
\hline 0,000 & 0,000 & 16,6 & $2,80 \mathrm{E}-04$ & $4,03 \mathrm{E}-01$ & $5,01 \mathrm{E}+11$ & $2,00 \mathrm{E}-10$ \\
\hline 0,188 & 0,765 & 16,6 & 2,94E-03 & $3,64 \mathrm{E}+00$ & $4,97 \mathrm{E}+11$ & $2,01 \mathrm{E}-10$ \\
\hline 0,000 & 0,000 & 28,5 & $1,40 \mathrm{E}-04$ & $1,06 \mathrm{E}+00$ & $6,01 \mathrm{E}+11$ & $5,70 \mathrm{E}-12$ \\
\hline 0,188 & 0,765 & 28,5 & $7,14 \mathrm{E}-03$ & $7,12 \mathrm{E}-01$ & $5,80 \mathrm{E}+11$ & $1,08 \mathrm{E}-10$ \\
\hline 0,000 & 0,000 & 37,5 & $1,40 \mathrm{E}-05$ & $2,63 \mathrm{E}-01$ & $1,06 \mathrm{E}+09$ & $1,08 \mathrm{E}-10$ \\
\hline 0,188 & 0,765 & 37,5 & $9,51 \mathrm{E}-03$ & $2,82 \mathrm{E}-02$ & $1,88 \mathrm{E}+09$ & $3,56 \mathrm{E}-08$ \\
\hline 0,000 & 0,000 & 44,4 & $2,80 \mathrm{E}-04$ & $5,91 \mathrm{E}-01$ & $8,51 \mathrm{E}+10$ & $2,38 \mathrm{E}-10$ \\
\hline 0,188 & 0,765 & 44,4 & $1,43 \mathrm{E}-02$ & $3,72 \mathrm{E}+00$ & $8,96 \mathrm{E}+10$ & $5,40 \mathrm{E}-10$ \\
\hline 0,000 & 0,000 & 100 & $4,20 \mathrm{E}-04$ & $3,29 \mathrm{E}-01$ & $1,57 \mathrm{E}+09$ & $5,54 \mathrm{E}-10$ \\
\hline 0,188 & 0,765 & 100 & $1,08 \mathrm{E}-01$ & $4,34 \mathrm{E}-01$ & $2,24 \mathrm{E}+09$ & $3,02 \mathrm{E}-08$ \\
\hline
\end{tabular}


Tabela 5: Correlação entre as variáveis.

\begin{tabular}{c|c|c|c}
\hline Variáveis & Tempo $(\mathbf{h})$ & Quartzo $(\%)$ & Pressão (MPa) \\
\hline Tempo $(\mathrm{h})$ & 1,00 & 0,00 & 0,95 \\
\hline Quartzo $(\%)$ & 0,00 & 1,00 & 0,01 \\
\hline Pressão $(\mathrm{MPa})$ & 0,95 & 0,01 & 1,00 \\
\hline Carga $(\mathrm{pF})$ & 0,34 & 0,69 & 0,33 \\
\hline $\mathrm{E}(\mathrm{V} . \mathrm{cm})$ & 0,18 & $-0,55$ & 0,13 \\
\hline$\rho(\Omega . c m)$ & 0,00 & $-0,69$ & 0,00 \\
\hline $\mathrm{J}(\mathrm{A} / \mathrm{cm} 2)$ & 0,00 & 0,52 & $-0,03$ \\
\hline
\end{tabular}

Assim, entre as variáveis estudadas, seria possível a construção de um modelo da carga acumulada como função do tempo, da quantidade de quartzo e da pressão, mostrados nas Figuras 3 e 4 . Outras possíveis combinações não são apresentadas devido à baixa correlação entre os dados experimentais e a superfície de resposta obtida.

As Figuras 3 (a) e (b) mostram que o aumento do tempo e da pressão, sempre em conjunto com o aumento da quantidade de quartzo, produzem um acréscimo na capacidade de armazenamento de carga elétrica do material. Assim, os resultados demonstram a natureza capacitiva dos materiais produzidos, que acumulam mais e mais carga elétrica com o passar do tempo. Além disso, as maiores cargas elétricas registradas ocorrem nos materiais contendo maiores quantidades de quartzo, indicando que esses materiais deveriam ser usados preferencialmente para essa aplicação sensora.

(a)
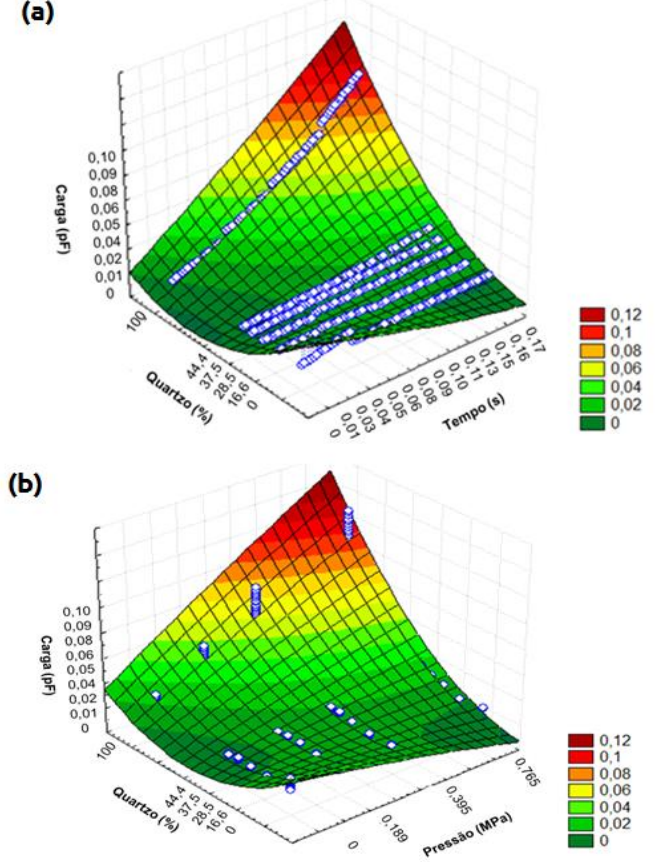

Figura 3: (a)Superfície de resposta da carga elétrica em função da quantidade de quartzo e do tempo $\left(R^{2}=0,910\right)$ e $(b)$ Superfície de resposta da carga elétrica em função da quantidade de quartzo e da pressão $\left(R^{2}=0,960\right)$. 


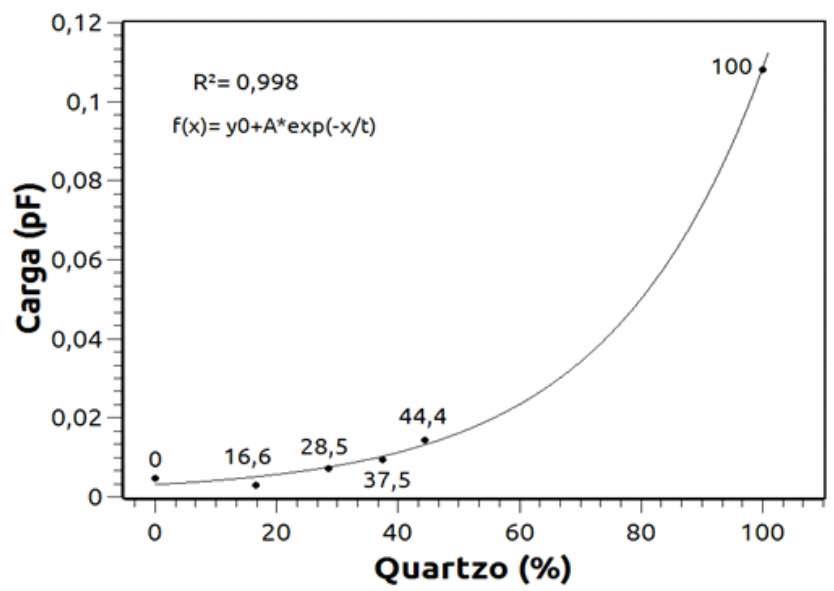

Figura 4: Aumento do armazenamento de carga em função da quantidade de quartzo nos compósitos.

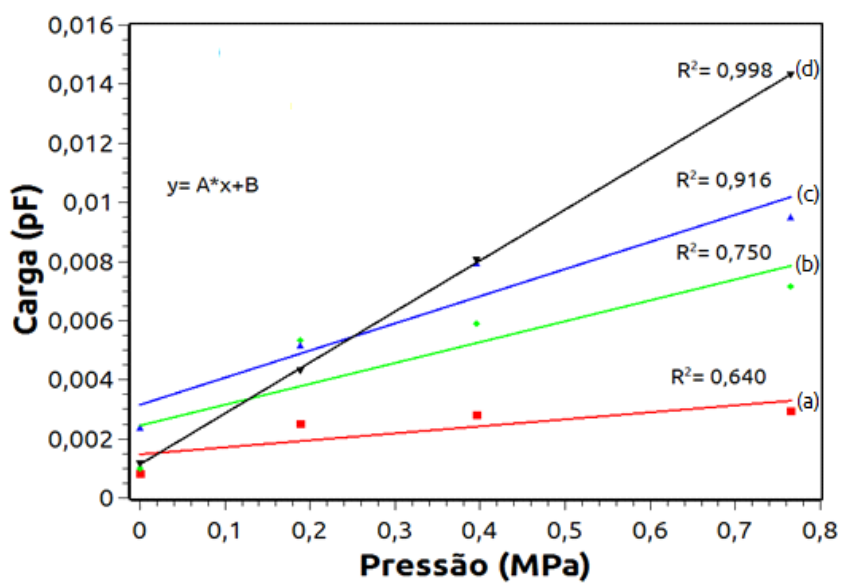

Figura 5: Aumento do armazenamento de carga em função da pressão aplicada nos compósitos (a) 16,6\%; (b) 28,5\%; (c) $37,5 \%$ e (d) $44,4 \%$ de quartzo.

A partir dos resultados da superfície de resposta mostrada na Figura 3 (a), foi possível construir a Figura 4. Esta figura permite observar a influência da quantidade de quartzo no armazenamento de carga dos materiais e no tempo final do experimento. A curva obtida apresentou crescimento exponencial da capacidade de armazenamento de carga com o aumento da quantidade de quartzo usada na matriz polimérica. Por sua vez, a análise da Figura 3 (b), permitiu a construção da Figura 5. Essa figura mostra a influência da pressão sobre o armazenamento de carga dos compósitos contendo $16,6 \%, 28,5 \%, 37,5 \%$ e 44,4\% de quartzo. Todas as respostas foram avaliadas por regressão linear. Os parâmetros determinados via regressão são mostrados na Tabela 6.

Tabela 6: Influência da quantidade de quartzo nas propriedades elétricas.

\begin{tabular}{l|l|l|l}
\hline Quartzo $(\%)$ & Coeficiente Linear & Coeficiente angular & $\mathrm{R}^{2}$ \\
\hline 16,6 & $1,48 \times 10^{-3}$ & $2,37 \times 10^{-3}$ & 0,640 \\
\hline 28,5 & $2,45 \times 10^{-3}$ & $7,06 \times 10^{-3}$ & 0,750 \\
\hline 37,5 & $3,15 \times 10^{-3}$ & $9,19 \times 10^{-3}$ & 0,916 \\
\hline 44,4 & $1,13 \times 10^{-3}$ & $1,73 \times 10^{-2}$ & 0,998 \\
\hline
\end{tabular}

Os dados contidos na Tabela 6 mostram que o aumento da concentração de quartzo nos compósitos produz um aumento linear da declividade das retas $\left(\mathrm{R}^{2}=0,947\right)$. Esse comportamento comprova que quantidades maiores de quartzo produzem materiais com maiores sensibilidades à compressão (razão entre a variação da carga em pF e a variação da pressão em MPa) [18]. Além disso, foi possível notar um 
crescimento da linearidade, via análise dos valores de $\mathrm{R}^{2}$ mostrados na Tabela 6 , dessas relações lineares em função da quantidade de quartzo. Deste modo, a maior linearidade $\left(\mathrm{R}^{2}=0,998\right)$ foi obtida com o uso da maior quantidade de quartzo $(44,4 \%)$. Essas respostas lineares são muito desejadas para o uso do material como sensor de pressão [19].Ainda que tenham mostrado baixa capacidade de armazenamento de carga, esses compósitos podem ainda ser melhorados, com o uso de outras cargas, tais como, nanotubos de carbono [21] ou negro de fumo condutor [22]. Apesar disso, esses resultados são muito encorajadores, uma vez que demonstram que o reaproveitamento desse material polimérico pós-consumo gera um produto de baixo custo e sensível à compressão, o qual pode ser de grande utilidade na Construção Civil como, por exemplo, um pavimento inteligente para edificações.

\section{CONCLUSÕES}

Os resultados obtidos através das análises foram capazes de mostrar o comportamento dos compósitos frente a diferentes tratamentos. A alternativa de reciclagem da goma de mascar mostrou ser eficiente, uma vez que, a incorporação dela na matriz de SBR já possibilita seu uso, como mostraram os resultados reométricos. O uso do quartzo como carga na matriz da goma de mascar/SBR proporcionou o aumento da resistência do material, mostrado pela análise reométrica e, também foi possível obter um compósito capaz de armazenar carga elétrica. Esse material apresentou uma resposta elétrica diretamente correlacionada com a pressão aplicada, sendo um possível candidato a material base para um sensor capacitivo de pressão. Além da questão tecnológica, o presente trabalho demonstrou que é perfeitamente factível recuperar as amostras de goma de mascar pós-consumo. Esse resultado é muito encorajador do ponto de vista ambiental e será alvo de futuras investigações de nosso grupo.

\section{AGRADECIMENTOS}

Os autores agradecem as agências de fomento CAPES 001, CNPq 304500/2019-4, FAPERJ (CNE-2020) e FINEP pelo suporte financeiro e bolsas.

\section{BIBLIOGRAFIA}

[1] SILVA, T.D. "Uso de granulado de borracha em substituição parcial ao agregado miúdo na produção de tijolos ecológicos". RevistaMatéria, v. 22, n. 4, pp. 1-12, Apr. 2017.

[2] MACHIN, E.B., PEDROSO, D.T., CARVALHO, J.A. "Energetic valorization of waste tires".Renewable and Sustainable Energy Reviews, v. 68, pp. 306-315, Feb. 2017.

[3] SILVA, A.S., NACIF, G.C.L., PANZERA, T.H., et al.,"Incorporation of rubber wastes into thermorigid polymeric matrix composites”. Revista Matéria, v. 17, n. 4, pp. 1158-1165, Oct. 2012.

[4] Eco-Unifesp. Tempo de decomposição de alguns resíduos. Disponível em:

$<$ https://dgi.unifesp.br/ecounifesp/index.php?option=com_content\&view=article\&id=16\&Itemid=11\#: :text =5\%20anos\%3A,pode\%20durar\%20at\%C3\%A9\%20cinco\%20anos> Acesso em 03 jan. 2021.

[5] SILVA, D.F.D. Desenvolvimento de blendas de poliestireno/composto de resíduo de borracha da indústria calçadista. Tese de D.Sc., Universidade Federal de Campina Grande, Campina Grande, Brasil, 2018.

[6] CANAZZA, M.A. Reciclagem de elastômeros via micro-ondas. Tese de D.Sc., Universidade de São Paulo, São Paulo, Brasil, 2019.

[7] SOUZA, J.P.S., DE MORAES, S.M., DODE, A.C. "Estudodas possibilidades de reciclagem da goma de mascar: estudo de caso no centro universitário metodista Izabela Hendrix (CEUNIH), BELO HORIZONTE, MG”. Revista Eletrônica de Gestão e Tecnologias Ambientais, v. 5, n. 1, p. 1-12, 2017.

[8] SEIFERT, K., ZAGRODNIK, R., STODOLNY, M., et al., "Biohydrogen production from chewing gum manufacturing residue in a two-step process of dark fermentation and photofermentation". Renewableenergy, v. 122, p. 526-532, 2018.

[9] Green-me. Na argentina chicletes se transformam em botas, chinelos e capas para celular e muito mais. GumPoint. Disponível em: <https://www.greenme.com.br/informarse/59356-na-argentina-chicletes-setransformam-em-botas-chinelos-capas-para-celular-e-muito-mais/> Acesso em 03 jan. 2021

[10] KROEGER, P.D.The feasibility of using monochloramine in recycling vulcanized styrene butadiene rubber. Tese de D.Sc., Universityof Louisville, Kentucky, EUA, 2013. 
[11] SANTOS, E., SILVA, L. Sensor inteligente miniaturizado de alta resolução para monitoramento semidistribuído de grandezas físicas com alta resolução e seu método construtivo. Depositante: Universidade Federal de Pernambuco; BR 1020170153630, Depósito: 18 julho 2017. Disponível em: https://attena.ufpe.br/bitstream/123456789/35410/1/BR\%2010\%202017\%20015363\%200\%20\%20870170050164\%20-\%20COA\%2030.pdf. Acesso em: 07 fev. 2021.

[12] LIU, Z., QIAN, Z., SONG, J.,et al., “Conducting and stretchable composites using sandwiched graphenecarbon nanotube hybrids and styrene-butadiene rubber". Carbon, v. 149, pp. 181-189, 2019.

[13] ARENHART, R.G. "Método de previsão de limiar de percolação e condutividade elétrica em compósitos poliméricos por simulação de Monte Carlo". TeseM.Sc., Universidade Federal de Santa Catarina, Santa Catarina, Brasil, 2012.

[14] JUNIOR, H.M.A., NUNES, R.C., VISCONTE, L.L. "Misturas NR/SBR: Influência da Composição e do Modo de Preparação Sobre Propriedades Mecânicas e Reométricas". Polímeros: Ciência e Tecnologia, v. 20, n.1, pp. 1-5, Mar. 2010.

[15] SOUZA, F.G., MICHEL, R.C., SOARES, B.G. "A methodology for studying the dependence of electrical resistivity with pressure in conducting composites". Polymer testing, v. 24, n. 8, p. 998-1004, Dec. 2005.

[16] AFIFI, A.A., AZEN, S.P.A..Statistical analysis: a computer oriented approach, 2 ed,New York, Academic press, 2014.

[17] OLIVEIRA, I.T., PACHECO, É.B., VISCONTE, L.L., et al.,Efeito de um novo acelerador de vulcanização nas propriedades reométricas de composições de borracha nitrílica com diferentes teores de AN. Polímeros: Ciência e Tecnologia, v. 20, n. Especial, 2010

[18] SILVA, L.M. A Fabricação de pneus com sílica. Tese M.Sc. Fundação Centro Universitário da Zona Oeste do Rio de Janeiro, Campo Grande, RJ, Brasil, 2013.

[19] AHMED, M.A., KANDIL, U.F., SHAKER, N.O., et al.,"The overall effect of reactive rubber nanoparticles and nano clay on the mechanical properties of epoxy resin". Journal of Radiation Research and Applied Sciences, v. 8, n. 4, pp. 549-561, Jun. 2015.

[20] RIBEIRO, M. Estudo das Propriedades Estruturais e Ópticas em Materiais Nanoestruturados a Base de Silício. Tese de D.Sc., Universidade de São Paulo, São Paulo, SP, Brasil, 2009.

[21] DUTRA, R.C.L., DINIZ, M.F., RIBEIRO, A.P.,et al."Determinação do teor de NR/SBR em misturas: associação de dados DTG e FT-IR". Polímeros: Ciência e Tecnologia, v.14, n.5, pp. 334-348, Oct. 2004.

[22] LAGO, R.M., GOMES, J.P.D.M., SANTOS, E.J.M., et al."Pyrolysis of rubber wastes from the mining industry for the production of fuels: pilot scale studies". Polímeros: Ciência e Tecnologia, v. 27, n. Suppl, p. 42-47, Mar. 2017.

[23] KANG, M.C., RIM, C.S., PAK, Y.T., et al."A simple analysis to improve linearity of touch mode capacitive pressure sensor by modifying shape of fixed electrode". SensorsandActuators A: Physical, v. 263, pp. 300-304, Aug. 2017.

[24] SAIGUSA, Y. "Quartz-based piezoelectric materials". In: Advanced Piezoelectric Materials, v.1, Woodhead Publishing, River Eletec Corporation, Nirasaki, Japão, pp. 197-233, 2017

\section{ORCID}

Fabíola da Silveira Maranhão Daniela Marques do Nascimento Fernando Gomes de Souza Junior Thuanny Moraes de Almeida Thiago Castro Lopes Tamara Nunes da Cunha Moreira Leila Y. Visconte

Romildo Dias https://orcid.org/0000-0002-9480-9294

https://orcid.org/0000-0003-2200-6609

https://orcid.org/0000-0001-5653-3939

https://orcid.org/0000-0001-8501-8864

https://orcid.org/0000-0003-0418-9202

https://orcid.org/0000-0003-3941-0974

https://orcid.org/0000-0001-8408-1036

https://orcid.org/0000-0001-5867-4452 